\title{
PARTIAL TRANSPHYSEAL ANTERIOR CRUCIATE LIGAMENT RECONSTRUCTION: CLINICAL, FUNCTIONAL, AND RADIOGRAPHIC OUTCOMES
}

\author{
Nirav K. Pandya, MD'1, Christina Allen, MD², Emily Monroe, MD², Caitlin Chambers, \\ $\mathrm{MD}^{4}$
}

${ }^{1}$ UCSF, Oakland, CA, USA, ${ }^{2}$ UCSF, USA, ${ }^{3}$ UCSF, USA, ${ }^{4}$ UCSF, USA

\section{BACKGROUND}

Partial transphyseal anterior cruciate ligament $(A C L)$ reconstruction is a technique utilized in the skeletally immature population. The femoral tunnel is placed in the distal femoral epiphysis whereas the tibial tunnel is placed in a transphyseal fashion medial to the tibial tubercle. This technique was introduced in an effort to lessen insult to the distal femoral physis of skeletally immature adolescents while also avoiding the technical difficulty of placing an intra-epiphyseal tunnel in the proximal tibia which at times can be non-anatomic. There is limited literature examining this technique. In this study we analyzed the concurrent surgical procedures, reoperation and graft failure rates, and radiographic outcomes in adolescents undergoing partial transphyseal ACL reconstruction.

\section{METHODS}

Consecutive patients undergoing partial transphyseal ACL reconstruction by the two senior authors (NP and CA) were retrospectively reviewed. Inclusion criteria consisted of patients with symptomatic ACL rupture with open distal femoral physes and at least two years of growth remaining by chronologic and physiologic age as determined by growth and pubertal history. All patients received hamstring autograft. Femoral tunnels were drilled in an intra-epiphyseal location utilizing small angle guides under fluoroscopic guidance. Transphyseal tibial tunnels were drilled in standard fashion with a tip-aiming guide while minimizing thermal damage from slow reaming, avoiding horizontal tunnel placement, and using extraphyseal graft fixation to lessen insult to the proximal tibial physis. Radiographic outcomes including bilateral limb length (LL) and alignment as judged by mechanical axis deviation (MAD), mechanical lateral distal femoral angle (mLDFA), and medial proximal tibial angle (MPTA) were measured on long standing anterior-to-posterior (AP) view radiographs. Growth disturbance was defined as $=1 \mathrm{~cm}$ leg length discrepancy, $=1 \mathrm{~cm}$ difference in MAD, or 5-degree difference in mLDFA or MPTA as compared to the non-operative side and MAD/mLDFA/MPTA outside of established range of normal values. Clinical outcomes including graft failure and need for repeat operation were recorded at each follow-up visit. Operative extremity alignment measurements were compared to non-operative extremity measurements utilizing a paired students t-test.

\section{RESULTS}

Twenty-four patients with average follow up of $31.5 \pm 17.1$ months met inclusion criteria for this study. Five female and 19 male patients were enrolled consisting of 13 left and 11 right knees. Average age at time of surgery was $12.3 \pm 0.9$ years (10.1-13.8 years). The average ACL graft size was $7.8 \mathrm{~mm} \pm 0.5 \mathrm{~mm}(6-9 \mathrm{~mm})$. Ten patients $(41.7 \%)$ had concurrent meniscal surgery, with seven $(29.2 \%)$ undergoing partial lateral meniscectomy, two $(8.3 \%)$ lateral meniscus repairs, and one patient $(4.2 \%)$ with medial and lateral meniscal repairs. Six patients $(25.0 \%)$ required reoperation at an average of $29.2 \pm 17.3$ months (1.5-49.5 months) for removal of hardware $(n=3)$, revision $A C L$ reconstruction $(n=2)$, and meniscus surgery $(n=1)$. Two patients had $A C L$ graft failure $(8.3 \%)$ during sporting activity and underwent revision ACL reconstruction at 19.7 months and 49.5 months post-operatively. There were no contralateral ACL tears. As shown in Table 1, comparison of the mean alignment and limb length measurements between all patients' operative to nonoperative extremity revealed no significant difference in femur length, MAD, MLDFA, or MPTA. There was a small but statistically significant difference in operative versus non-operative tibia length $(390.2 \mathrm{~cm}$ versus $392.4 \mathrm{~cm}, \mathrm{p}=0.0004)$ and limb length $(880.9 \mathrm{~cm}$ versus $884.0 \mathrm{~cm}$, $\mathrm{p}=0.02)$. In analyzing individual patients' limb length or alignment differences, five patients $(20.8 \%)$ were identified with a growth disturbance. One patient had isolated shortening of the operative extremity, two with significant lateral deviation of the MAD, and two with both shortening and

\footnotetext{
This open-access article is published and distributed under the Creative Commons Attribution - NonCommercial - No Derivatives License (http://creativecommons.org/licenses/by-nc-nd/4.0/), which permits the noncommercial use, distribution, and reproduction of the article in any medium, provided the original author and source are credited. You may not alter, transform, or build upon this article without the permission of the Author(s). For article reuse guidelines, please visit SAGE's website at http://www.sagepub.com/journals-permissions.
} 
lateral MAD translation. No patients had significant side-to-side difference in mLDFA or MPTA. Femoral shortening accounted for the majority of the limb length discrepancy in two of the three patients with significant limb length discrepancy. All patients returned to sport.

\section{CONCLUSIONS / SIGNIFICANCE}

Partial transphyseal $A C L$ reconstruction has a $25.0 \%$ re-operation rate, most often for hardware removal, and an $8.3 \%$ graft failure rate. Overall, approximately $20 \%$ of patients undergoing partial transphyseal ACL reconstruction had a growth disturbance but none required surgical intervention for these disturbances. While the partial transphyseal technique spares the distal femoral physis, femur-dominant limb length discrepancy can still occur. Drilling and graft placement across the tibial physis appears to be safe. Further studies are needed to directly compare the radiographic, functional, and clinical outcomes of partial transphyseal ACL reconstruction with transphyseal and all-epiphyseal techniques in the pediatric population.

\begin{tabular}{|lllr|}
\multicolumn{4}{|c|}{ Table 1: Operative vs Non-Operative Limb Measurements } \\
& $\begin{array}{c}\text { Operative } \\
\text { (mean } \pm \text { SD) }\end{array}$ & \multicolumn{1}{c|}{$\begin{array}{c}\text { Non-Operative } \\
\text { (mean } \pm \text { SD) }\end{array}$} & $\begin{array}{c}\text { p- } \\
\text { value }\end{array}$ \\
Limb Length $(\mathrm{cm})$ & $490.7 \pm 33.8$ & $491.6 \pm 33.3$ & 0.4 \\
Femur Length & $390.2 \pm 25.3$ & $392.4 \pm 25.2$ & $\mathbf{0 . 0 0 0 4}$ \\
Tibia Length & $880.9 \pm 57.9$ & $884 \pm 57.7$ & $\mathbf{0 . 0 2}$ \\
MAD (mm) & $5.4 \pm 9.1$ & $5.8 \pm 6.4$ & 0.8 \\
MLDFA (degrees) & $85.8 \pm 2.6$ & $86 \pm 2.6$ & 0.8 \\
MPTA (degrees) & $88.2 \pm 1.8$ & $87.9 \pm 2.1$ & 0.44
\end{tabular}

The Orthopaedic Journal of Sports Medicine, 7(3)(suppl 1)

DOI: $10.1177 / 2325967119500081$

(CThe Author(s) 2019 